\title{
The Eichler Commutation Relation for theta series with spherical harmonics
}

\author{
by \\ Lynne H. Walling (Boulder, Colo.)
}

It is well known that classical theta series which are attached to positive definite rational quadratic forms yield elliptic modular forms, and linear combinations of theta series attached to lattices in a fixed genus can yield both cusp forms and Eisenstein series whose weight is one-half the rank of the quadratic form. In contrast, generalized theta series - those augmented with a spherical harmonic polynomial — will always yield cusp forms whose weight is increased by the degree of the spherical harmonic. A recent demonstration of the far-reaching importance of generalized theta series is Hijikata, Pizer and Shemanske's solution to Eichler's Basis Problem [4] (cf. [2]) in which character twists of such theta series are used to provide a basis for the space of newforms.

In this paper we consider theta series with spherical harmonics over a totally real number field. We show that such theta series are Hilbert modular cusp forms whose weight is integral or half-integral, depending on the rank of the associated lattice. We explicitly describe the action of the Hecke operators on these theta series in terms of other theta series, yielding a generalization of the well-known Eichler Commutation Relation. Finally, we use these theta series to construct Hilbert modular forms which are invariant under a subalgebra of the Hecke algebra. We are able to show that if the quadratic form has rank $m$ and the spherical harmonic has degree $l$, then the theta series attached to the genus of a lattice is identically zero whenever $l$ is small relative to $m$; in particular, the associated collection of theta series are linearly dependent.

1. The transformation formula. Here we derive the transformation formula for theta series with spherical harmonics. Our approach is similar to that of Eichler in [3], but unlike the derivation in [3], we first derive an

Partially supported by NSF DMS 9103303. 
inversion formula by identifying our theta series with a Siegel modular form.

Let $\mathbb{K}$ be a totally real number field of degree $n$ over $\mathbb{Q}$, and let $\mathcal{O}$ and $\partial$ denote its ring of integers and its different (respectively). Let $V$ be an $m$ dimensional vector space over $\mathbb{K}$ with $B: V \times V \rightarrow \mathbb{K}$ a symmetric bilinear form such that $Q(x) \stackrel{\text { def }}{=} B(x, x)$ is totally positive for all nonzero $x \in V$. Take $L$ to be a lattice on $V$ (so $L$ is a rank $m \mathcal{O}$-module contained in $V$ ). We let $L^{\#}$ denote the dual of $L$ (i.e. $L^{\#}=\{x \in V: B(x, L) \subseteq \mathcal{O}\}$ ) and $\mathbf{n} L$ the norm of $L$ (i.e. $\mathbf{n} L$ is the fractional $\mathcal{O}$-ideal generated by $\frac{1}{2} Q(L)$ ). Then as shown in [3] (cf. [7]), $(\mathbf{n} L)^{-1}\left(\mathbf{n} L^{\#}\right)^{-1}$ is an integral ideal. For later convenience, we take $\mathcal{I}_{L}$ to be the smallest fractional ideal of $\mathcal{O}$ such that $\mathcal{I}_{L}^{2} \supseteq \mathbf{n} L$, and we put $N(L)=\mathbf{n} L$ in case $m$ is even, and $N(L)=\mathcal{I}_{L}^{2}$ in case $m$ is odd. We also put $\mathcal{N}$ equal to the stufe of $L$ as defined in [7]. Thus if $m$ is even, $\mathcal{N}$ is the product of $(\mathbf{n} L)^{-1}\left(\mathbf{n} L^{\#}\right)^{-1}$ and perhaps some dyadic primes; if $m$ is odd, $\mathcal{N}=\left(\mathbf{n} L^{\#}\right)^{-1} \mathcal{I}_{L}^{-2}$.

Viewing $L$ as a $\mathbb{Z}$-module, we take $\left\{\lambda_{1}, \ldots, \lambda_{m n}\right\}$ to be a $\mathbb{Z}$-basis for $L$. Having fixed a $\mathbb{K}$-basis $\left\{v_{1}, \ldots, v_{m}\right\}$ for $V$, we associate vectors of $V$ with $m \times 1$ column vectors whose entries lie in $\mathbb{K}$; hence for $T=\left(\lambda_{1} \ldots \lambda_{m n}\right) \in$ $M_{m n, m}(\mathbb{K}), L=T \mathbb{Z}^{m n}$. We remark here that the matrix

$$
\left(\begin{array}{c}
T^{(1)} \\
\vdots \\
T^{(n)}
\end{array}\right)
$$

is invertible (where the superscript of $(j)$ denotes the image under the $j$ th embedding of $\mathbb{K} / \mathbb{Q}$ into $\mathbb{R})$. To see this, notice that $\langle v, w\rangle=\operatorname{Tr}\left({ }^{t} v \cdot w\right)$ is a symmetric bilinear form on the rational vector space $V$ (here we still identify $v$ and $w$ with $m \times 1$ column vectors). Let $\left\{\kappa_{1}, \ldots, \kappa_{m n}\right\}$ be a dual basis for $\left\{\lambda_{1}, \ldots, \lambda_{m n}\right\}$ with respect to $\langle *, *\rangle$ (see [5]); thus

and so

$$
\left\langle\lambda_{i}, \kappa_{j}\right\rangle= \begin{cases}1 & \text { if } i=j, \\ 0 & \text { otherwise }\end{cases}
$$

$$
\left(\begin{array}{ccc}
{ }^{t} \kappa_{1}^{(1)} & \ldots & { }^{t} \kappa_{1}^{(n)} \\
& \ddots & \\
{ }^{t} \kappa_{m n}^{(1)} & \ldots & { }^{t} \kappa_{m n}^{(n)}
\end{array}\right)\left(\begin{array}{ccc}
\lambda_{1}^{(1)} & \ldots & \lambda_{m n}^{(1)} \\
& \ddots & \\
\lambda_{1}^{(n)} & \ldots & \lambda_{m n}^{(n)}
\end{array}\right)=\left(\left\langle\kappa_{i}, \lambda_{j}\right\rangle\right)=I_{m n} .
$$

Set $F=\left(B\left(v_{i}, v_{j}\right)\right)$; then since $Q$ is a totally positive quadratic form,

$$
Z \stackrel{\text { def }}{=} \sum_{j=1}^{n}\left({ }^{t} T F T\right)^{(j)} z_{j}=\left({ }^{t} T^{(1)} \ldots{ }^{t} T^{(n)}\right)\left(\begin{array}{lll}
\ddots & & \\
& F^{(j)} z_{j} & \\
& & \ddots
\end{array}\right)\left(\begin{array}{c}
T^{(1)} \\
\vdots \\
T^{(n)}
\end{array}\right)
$$

is in Siegel upper half-space whenever $z=\left(z_{1}, \ldots, z_{n}\right) \in \mathcal{H}^{n}$ (where $\mathcal{H}$ denotes the complex upper half-plane). Given $w=\left(w_{1}, \ldots, w_{n}\right)$ with $w_{j} \in$ 
$\mathbb{C}^{m}$, we can find some $u \in \mathbb{C}^{m n}$ such that ${ }^{t}\left(T^{(1)} \ldots T^{(n)}\right) u={ }^{t}\left(w_{1} \ldots w_{n}\right)$. Let $Z\langle\langle x\rangle\rangle$ denote ${ }^{t} x Z x$; then for any $x \in \mathbb{Q}^{m n}$ with $v=T x \in V$,

$$
Z\langle\langle x+u\rangle\rangle=\sum_{j=1}^{n}\left({ }^{t} T F T\right)^{(j)}\langle\langle x+u\rangle\rangle z_{j}=\sum_{j=1}^{n} F^{(j)}\left\langle\left\langle v^{(j)}+w_{j}\right\rangle\right\rangle z_{j} ;
$$

note that if $w_{0} \in V$ and $w_{j}=w_{0}^{(j)}$ then

$$
Z\langle\langle x+u\rangle\rangle=\operatorname{Tr}\left(Q\left(v+w_{0}\right) z\right) .
$$

Set $\vartheta(Z ; u)=\sum_{x \in \mathbb{Z}^{m n}} \exp \{\pi i Z\langle\langle x+u\rangle\rangle\}$; by Lemma 1.3.1 of [1] we have

$$
(\operatorname{det}(-i Z))^{1 / 2} \vartheta(Z ; u)=\sum_{x \in \mathbb{Z}^{m n}} \exp \left\{\pi i\left(-Z^{-1}\langle\langle x\rangle\rangle+2{ }^{t} x u\right)\right\}
$$

where the square-root function is defined as in the above cited lemma. Given our choice of $Z$, we find that

$$
\operatorname{det}(-i Z)=(-i)^{m n / 2} z^{m / 2} \sqrt{\Phi(L)}
$$

where

$$
z^{m / 2}=\prod_{j=1}^{n} z_{j}^{m / 2} \quad \text { and } \quad \Phi(L)=\operatorname{det}\left(\sum_{j=1}^{n}\left({ }^{t} T F T\right)^{(j)}\right) .
$$

Also,

$$
\begin{aligned}
Z^{-1} & =\sum_{j=1}^{n}\left({ }^{t} S F^{-1} S\right)^{(j)} \frac{1}{z_{j}} \\
& =\left({ }^{t} S^{(1)} \ldots{ }^{t} S^{(n)}\right)\left(\begin{array}{lll}
\ddots & \\
& \left(F^{(j)}\right)^{-1} \frac{1}{z_{j}} & \\
& & \ddots
\end{array}\right)\left(\begin{array}{c}
S^{(1)} \\
\vdots \\
S^{(n)}
\end{array}\right)
\end{aligned}
$$

where $S=\left(\kappa_{1} \ldots \kappa_{m n}\right)$; thus

$$
\begin{aligned}
\sum_{x \in \mathbb{Z}^{m n}} \exp \left\{\pi i\left(-Z^{-1}\left\langle\langle x\rangle+2{ }^{t} x u\right)\right\}\right. & \\
= & \sum_{x \in \mathbb{Z}^{m n}} \exp \left\{\pi i\left(-Z^{-1}\langle\langle x\rangle\rangle+2{ }^{t} x\left({ }^{t} S^{(1)} \ldots{ }^{t} S^{(n)}\right)\left(\begin{array}{c}
w_{1} \\
\vdots \\
w_{n}
\end{array}\right)\right)\right\} \\
= & \sum_{x \in S \mathbb{Z}^{m n}} \exp \left\{\pi i\left(\sum_{j=1}^{n}-\frac{1}{z_{j}}\left(F^{-1}\right)^{(j)}\left\langle\left\langle x^{(j)}\right\rangle\right\rangle+2{ }^{t} x^{(j)} w_{j}\right)\right\} \\
= & \sum_{x \in F^{-1} S \mathbb{Z}^{m n}} \exp \left\{\pi i\left(\sum_{j=1}^{n}-\frac{1}{z_{j}} F^{(j)}\left\langle\left\langle x^{(j)}\right\rangle\right\rangle+2{ }^{t} x^{(j)} F^{(j)} w_{j}\right)\right\} .
\end{aligned}
$$


Notice that $F^{-1} S \mathbb{Z}^{m n}$ is $\widetilde{L}$, the complement of $L$, which is the lattice dual to $L$ with respect to the bilinear form $\operatorname{Tr}_{\mathbb{K} / \mathcal{Q}} \circ B$. Then with a slight abuse of notation, formula (1) gives us the "inversion formula":

$$
\theta(L, w ; z)=\frac{i^{m n / 2}}{z^{m / 2} \sqrt{\Phi(L)}} \sum_{y \in \widetilde{L}} e\left\{-\frac{1}{z} Q(y)+2 B(y, w)\right\}
$$

where $\theta(L, w ; z)=\sum_{x \in L} e\{Q(x+w) z\}$ and $e\{\alpha z\}$ means $\exp \{\pi i \operatorname{Tr}(\alpha z)\}$. (When $w \notin V$ we identify $x$ and $w$ with column vectors and interpret $Q(x+w)$ as $F\langle\langle x+w\rangle\rangle$.

From this inversion formula we derive an inversion formula for inhomogeneous theta series with spherical harmonics. Now, a degree $l \quad\left(l \in \mathbb{Z}_{+}\right)$ spherical harmonic $P$ relative to the lattice $L$ is a linear combination of functions on $L$ of the form $P_{\zeta, l}(x)=\prod_{j=1}^{n}\left(x^{(j)} F^{(j)} \zeta_{j}\right)^{l}$ where $\zeta_{j} \in \mathbb{C}^{m}$ such that ${ }^{t} \zeta_{j} F^{(j)} \zeta_{j}=0$ (here $x$ is again identified with an $m \times 1$ column vector using the basis $\left\{v_{1}, \ldots, v_{m}\right\}$ for $\left.V\right)$. Thus it suffices to derive an inversion formula with $P=P_{\zeta, l}$. To derive this formula, we take $w_{j}=v^{(j)}+\sigma_{j} \zeta_{j}$ where $v \in V, \sigma_{j}$ is a scalar, $\zeta_{j} \in \mathbb{C}^{m}$ and ${ }^{t} \zeta_{j} F^{(j)} \zeta_{j}=0$; we then differentiate equation (2) $l$ times with respect to each $\sigma_{j}$. Setting the $\sigma_{j}=0$ then gives us

$$
\begin{aligned}
z^{l} \sum_{x \in L} P_{w, l}(x+v) & \{Q(x+v) z\} \\
= & \frac{i^{m n / 2}}{z^{m / 2} \sqrt{\Phi(L)}} \sum_{y \in \widetilde{L}} P_{w, l}(y) e\left\{-\frac{1}{z} Q(y)+2 B(y, v)\right\} .
\end{aligned}
$$

Thus for any degree $l$ spherical harmonic $P$ relative to $L$ and the inhomogeneous theta series

$$
\theta(L, P, v ; z) \stackrel{\text { def }}{=} \sum_{x \in L} P(x+v) e\{Q(x+v) z\}
$$

we have

$$
\theta(L, P, v ; z)=\frac{i^{m n / 2}}{z^{m / 2+l} \sqrt{\Phi(L)}} \sum_{y \in \widetilde{L}} P(y) e\left\{-\frac{1}{z} Q(y)+2 B(y, v)\right\} .
$$

For $w_{j}=w_{0}^{(j)}$ with $w_{0} \in \widetilde{L}$ this is the inversion formula from [3]. Note, however, that $i$ has an exponent of $m n / 2$ and not $-m n / 2$; J. Stopple informs us that in carefully following Eichler's proof he found a minor oversight which accounts for this difference in sign.

Without loss of generality, we will assume herein that $l$ is even. For, if $l$ is odd then $\theta(L, P ; z) \stackrel{\text { def }}{=} \theta(L, P, 0 ; z)=0$ since the coefficient of $e\{2 \zeta z\}$ is $\sum_{x \in L, Q(x)=2 \zeta} P(x)$ and $P(x)+P(-x)=0$ when $l$ is odd. 
The arguments of [3] or of [7] can now be used to derive the transformation formula; for completeness, we will sketch here a simplified version of this proof. Take $\left(\begin{array}{ll}a & b \\ c & d\end{array}\right)$ such that $a, d \in \mathcal{O}, d \neq 0, b \in(N(L))^{-1} \partial^{-1}$, $c \in \mathcal{N} N(L) \partial$ and $a d-b c=1$. Then as in [7] we have

$$
\begin{aligned}
\theta\left(L, P ; \frac{a z+b}{c z+d}\right) \\
\quad=\sum_{x \in L} P(x) e\left\{Q(x) \frac{a z+b}{c z+d}\right\} \\
=\sum_{x_{0} \in L / d L} e\left\{\frac{b}{d} Q\left(x_{0}\right)\right\} \sum_{x \in d L} P\left(x+x_{0}\right) e\left\{\frac{Q\left(x+x_{0}\right)}{d(d / z+c)}\right\}
\end{aligned}
$$

and by $(3)$,

$$
\begin{aligned}
= & \frac{i^{m n / 2}}{\sqrt{\Phi(d L)}}(d(d / z+c))^{m / 2+l} \\
& \times \sum_{x_{0} \in L / d L} e\left\{\frac{b}{d} Q\left(x_{0}\right)\right\} \sum_{y \in d^{-1} \widetilde{L}} P(y) e\left\{-Q(y) d(d / z+c)+2 B\left(y, x_{0}\right)\right\} \\
= & \frac{i^{m n / 2}}{\sqrt{\Phi(L)}}(d / z+c)^{m / 2+l} N(d)^{l-m / 2} \\
& \times \sum_{x_{0} \in L / d L} e\left\{\frac{b}{d} Q\left(x_{0}\right)+\frac{2}{d} B\left(y, x_{0}\right)-\frac{c}{d} Q(y)\right\} N(d)^{-l} P(y) e\left\{-Q(y) \frac{1}{z}\right\}
\end{aligned}
$$

and since $\frac{b}{d} Q\left(x_{0}\right)+\frac{2}{d} B\left(y, x_{0}\right)-\frac{c}{d} Q(y) \equiv-\frac{c}{d} Q\left(b x_{0}+y\right)\left(\bmod 2 \partial^{-1}\right)$

$$
\begin{aligned}
= & \frac{i^{m n / 2}}{\sqrt{\Phi(L)}}(d / z+c)^{m / 2+l} N(d)^{-m / 2} \\
& \times \sum_{\substack{x_{0} \in L / d L \\
y \in \widetilde{L}}} e\left\{-\frac{c}{d} Q\left(b x_{0}+y\right)\right\} P(y) e\left\{-Q(y) \frac{1}{z}\right\} .
\end{aligned}
$$

Now, for any prime $\mathcal{P}$ dividing $d$ we must have

$$
-\operatorname{ord}_{\mathcal{P}} \mathcal{N} N(L) \partial=\operatorname{ord}_{\mathcal{P}} b \geq-\operatorname{ord}_{\mathcal{P}} N(L) \partial
$$

(since $c \in \mathcal{N} N(L) \partial, b \in(N(L))^{-1} \partial^{-1}$ and $a d-b c=1$ ); hence $\mathcal{P}$ cannot divide $\mathcal{N}$. Thus we can follow the argument of [7] to show that $b x_{0}+y$ runs 
over $\widetilde{L} / d \widetilde{L}$ as $x_{0}$ runs over $L / d L$. So

$$
\begin{aligned}
\theta\left(L, P ; \frac{a z+b}{c z+d}\right)= & \frac{i^{m n / 2}}{\sqrt{\Phi(L)}}\left(\frac{d}{z}+c\right)^{m / 2+l} N(d)^{-m / 2} \\
& \times \sum_{x_{0} \in L / d L} e\left\{-\frac{c}{d} Q\left(b x_{0}\right)\right\} \sum_{y \in \widetilde{L}} P(y) e\left\{-\frac{1}{z} Q(y)\right\}
\end{aligned}
$$

and by $(3)$,

$$
\begin{aligned}
= & \left(\frac{d}{z}+c\right)^{m / 2+l} z^{m / 2+l} N(d)^{-m / 2} \\
& \times \sum_{x_{0} \in L / d L} e\left\{\frac{b}{d} Q\left(x_{0}\right)\right\} \theta(L, P ; z) .
\end{aligned}
$$

The arguments used to prove Theorem 3.7 of [7] immediately give us

TheOREM 1. Let $Q$ be a totally positive quadratic form on the rank $m$ lattice $L$ and let $P$ be a degree $l$ spherical harmonic relative to $L$. Then $\theta(L, P ; z)$ is a Hilbert modular form of weight $m / 2+l$ and quadratic character $\chi_{L}$ which transforms under the group

$$
\left\{\left(\begin{array}{ll}
a & b \\
c & d
\end{array}\right) \in\left(\begin{array}{cc}
\mathcal{O} & (N(L))^{-1} \partial^{-1} \\
\mathcal{N} N(L) \partial & \mathcal{O}
\end{array}\right): a d-b c=1\right\} .
$$

Remark. Let us here make more precise the meaning of the theorem. When $m$ is odd then we must associate an automorphy factor with a matrix $A=\left(\begin{array}{ll}a & b \\ c & d\end{array}\right)$ in the above group of matrices. The automorphy factor we use is that defined in [7],

$$
\frac{\theta_{\mathcal{I}_{L}}(A z)}{\theta_{\mathcal{I}_{L}}(z)}, \quad \text { where } \theta_{\mathcal{I}_{L}}(z)=\sum_{\alpha \in \mathcal{I}_{L}} e\left\{2 \alpha^{2} z\right\}
$$

This theta function satisfies the transformation formula (2) (with stufe $4 \mathcal{O}$ ); hence

$$
\begin{aligned}
\theta(L, P ; z) \mid\left[A, \frac{\theta_{\mathcal{I}_{L}}(A z)}{\theta_{\mathcal{I}_{L}}(z)}\right] & \stackrel{\text { def }}{=}\left(\frac{\theta_{\mathcal{I}_{L}}(A z)}{\theta_{\mathcal{I}_{L}}(z)}\right)^{-m-2 l} \theta(L, P ; A z) \\
& =\chi_{L}(d) \theta(L, P ; z) .
\end{aligned}
$$

When $m$ is even then

$$
\theta(L, P ; z) \mid A \stackrel{\text { def }}{=}(c z+d)^{-m / 2-l} \theta(L, P ; A z)=\chi_{L}(d) \theta(L, P ; z) .
$$

Note that since $\chi_{L}$ is a quadratic character modulo $\mathcal{N}, \chi_{L}(d)=\chi_{L}(a)$. Also note that the theorem holds trivially for $l$ odd, for as remarked earlier, in that case $\theta(L, P ; z)=0$. 
To take advantage of theory already developed, we want Hilbert modular forms which transform under matrices

$$
\left(\begin{array}{ll}
a & b \\
c & d
\end{array}\right) \in\left(\begin{array}{cc}
\mathcal{O} & (N(L))^{-1} \partial^{-1} \\
\mathcal{N} N(L) \partial & \mathcal{O}
\end{array}\right)
$$

where $a d-b c$ is any totally positive unit (if $m$ is even) or the square of any unit (if $m$ is odd). Recall that we are assuming $l$ is even; so for $u \in \mathcal{U}=\mathcal{O}^{\times}$ we have

$$
\begin{aligned}
\theta(L, P ; z) \mid\left(\begin{array}{cc}
u^{2} & 0 \\
0 & 1
\end{array}\right) & =N\left(u^{2}\right)^{m / 4+l / 2} \sum_{x \in L} P\left(\frac{x}{u}\right) e\{Q(x) z\} \\
& =N\left(u^{2}\right)^{m / 4+l / 2} N(u)^{-l} \theta(L, P ; z)=\theta(L, P ; z)
\end{aligned}
$$

(since $N\left(u^{2}\right)=1, N(u)= \pm 1$ and $l$ is even). Thus $\theta(L, P ; z)$ transforms under all matrices

$$
\left(\begin{array}{ll}
a & b \\
c & d
\end{array}\right) \in\left(\begin{array}{cc}
\mathcal{O} & (N(L))^{-1} \partial^{-1} \\
\mathcal{N} N(L) \partial & \mathcal{O}
\end{array}\right)
$$

with $a d-b c \in \mathcal{U}^{2}$. Now consider the case where $m$ is even. In the case where $P=1$ (and $l=0$ ), we previously forced the transformation property we desire by considering $\sum_{u} \theta\left(L^{u} ; z\right)$ where the sum ran over a (finite) set of totally positive units $u$ such that the scaled lattices $L^{u}$ lay in distinct isometry classes (see [5]). When $P \neq 1$ and $L^{v} \simeq L$ via the map $\varphi_{v}: L^{v} \rightarrow L$, we have

$$
\begin{aligned}
\theta\left(L^{v}, P ; z\right) & =\sum_{x \in L} P(x) e\{v Q(x) z\}=\sum_{x \in L} P(x) e\left\{Q\left(\varphi_{v}^{-1} x\right) z\right\} \\
& =\sum_{x \in L} P\left(\varphi_{v} x\right) e\{Q(x) z\}=\theta\left(L, P \circ \varphi_{v} ; z\right) .
\end{aligned}
$$

(Note that since $\varphi_{v}: L^{v} \rightarrow L$ is an isometry, $\varphi_{v}$ is an automorphism of $L$ such that $Q\left(\varphi_{v} x\right)=Q^{v}(x)=v Q(x)$.) Letting

$$
\mathcal{U}_{L}=\left\{v \in \mathcal{U}^{+}: L^{v} \simeq L\right\}
$$

we see that

$$
\begin{aligned}
\frac{1}{\left[\mathcal{U}_{L}: \mathcal{U}^{2}\right]} \sum_{u \in \mathcal{U}^{+} / \mathcal{U}_{L}} \sum_{v \in \mathcal{U}_{L} / \mathcal{U}^{2}} & \theta\left(L^{u}, P \circ \varphi_{v} ; z\right) \\
= & \sum_{u \in \mathcal{U}^{+} / \mathcal{U}_{L}} \theta\left(L^{u}, \frac{1}{\left[\mathcal{U}_{L}: \mathcal{U}^{2}\right]} \sum_{v \in \mathcal{U}_{L} / \mathcal{U}^{2}} P \circ \varphi_{v} ; z\right)
\end{aligned}
$$

transforms under all matrices

$$
\left(\begin{array}{ll}
a & b \\
c & d
\end{array}\right) \in\left(\begin{array}{cc}
\mathcal{O} & (N(L))^{-1} \partial^{-1} \\
\mathcal{N} N(L) \partial & \mathcal{O}
\end{array}\right)
$$


with $a d-b c \in \mathcal{U}^{+}$. (One easily checks that $\sum_{v} P \circ \varphi_{v}$ is a spherical harmonic relative to $L^{u}$ for any $u \in \mathcal{U}^{+}$.) So when $m$ is even, we consider the modular form

$$
\theta([L],[P] ; z) \stackrel{\text { def }}{=} \sum_{u \in \mathcal{U}^{+} / \mathcal{U}_{L}} \theta\left(L^{u}, \frac{1}{\left[\mathcal{U}_{L}: \mathcal{U}^{2}\right]} \sum_{v \in \mathcal{U}_{L} / \mathcal{U}^{2}} P \circ \varphi_{v} ; z\right) ;
$$

so that we may simultaneously treat the case where $m$ is odd, we set

$$
\theta([L],[P] ; z)=\theta(L, P ; z)
$$

when $m$ is odd. So essentially $[L]$ is the union $\bigcup_{u} L^{u}$ where $u$ runs over $\mathcal{U}^{+} / \mathcal{U}_{L}$, and $[P]$ is the "smoothed" spherical harmonic relative to $L$. Thus we have the

Corollary. The function $\theta([L],[P] ; z)$ is a modular form of weight $m / 2+l$ with character $\chi_{L}$ and which transforms under the group

$$
\Gamma_{0}(\mathcal{N}, N(L))=\left\{A \in\left(\begin{array}{cc}
\mathcal{O} & (N(L))^{-1} \partial^{-1} \\
\mathcal{N} N(L) \partial & \mathcal{O}
\end{array}\right): \operatorname{det} A \in \mathcal{U}^{+}\right\}
$$

(when $m$ is even) or

$$
\widetilde{\Gamma}_{0}(\mathcal{N}, N(L))=\left\{\widetilde{A}: A \in\left(\begin{array}{cc}
\mathcal{O} & (N(L))^{-1} \partial^{-1} \\
\mathcal{N} N(L) \partial & \mathcal{O}
\end{array}\right), \operatorname{det} A \in \mathcal{U}^{2}\right\}
$$

(when $m$ is odd).

2. $\theta([L],[P] ; z)$ as a cusp form. We now verify that $\theta(L, P ; z)$ (and hence $\theta([L],[P] ; z))$ is a cusp form where we assume $P \neq 1$. The computation is very similar to that in Lemma 1 of [12]. It is clear that $\theta(L, P ; z)$ is 0 at the cusp $i \infty$. For any $\beta \in \mathbb{K}^{\times}$, we write $\beta=\alpha / \gamma$ where $\alpha, \gamma \in \mathcal{O}$ and $\alpha \gg 0$; we want to show that

$$
\lim _{z \rightarrow i \infty} \theta(L, P ; z) \mid A=0
$$

where $A=\left(\begin{array}{ll}\alpha & 0 \\ \gamma & 1\end{array}\right)$. When $m$ is odd we must associate with $A$ some automorphy factor; our computation is independent of this automorphy factor, though, for changing our choice of automorphy factor can only change the value of $\theta(L, P ; z) \mid A$ by a fourth root of unity (cf. [12]). Now,

$$
\theta(L, P ; A z)=\sum_{x \in L} P(x) e\left\{Q(x) \frac{\alpha}{\gamma+1 / z}\right\}
$$

and by (3),

$$
=\frac{i^{m n / 2}}{\sqrt{\Phi(L)}}\left(\frac{\gamma+1 / z}{\alpha}\right)^{m / 2+l} \sum_{x \in \widetilde{L}} P(x) e\left\{-Q(x) \frac{\gamma+1 / z}{\alpha}\right\}
$$




$$
\begin{aligned}
= & \frac{i^{m n / 2}}{\sqrt{\Phi(L)}}\left(\frac{\gamma+1 / z}{\alpha}\right)^{m / 2+l} \\
& \times \sum_{\substack{x_{0} \in \widetilde{L} / \alpha L \\
x \in \alpha L}} e\left\{-\frac{\gamma}{\alpha} Q\left(x_{0}\right)\right\} P\left(x+x_{0}\right) e\left\{-Q\left(x+x_{0}\right) \frac{1}{\alpha z}\right\} \\
= & \frac{i^{m n / 2}}{\sqrt{\Phi(L)}}\left(\frac{\gamma+1 / z}{\alpha}\right)^{m / 2+l} \\
& \times \sum_{\substack{x_{0} \in \widetilde{L} / \alpha L \\
x \in \alpha L}} e\left\{-\frac{\gamma}{\alpha} Q\left(x_{0}\right)\right\} \theta\left(\alpha L, P, x_{0} ;-1 / \alpha z\right)
\end{aligned}
$$

and again by (3),

$$
\begin{aligned}
= & \frac{i^{m n}}{N(\alpha)^{m} \Phi(L)}\left(\frac{\gamma+1 / z}{\alpha}\right)^{m / 2+l} \sum_{x_{0} \in \widetilde{L} / \alpha L} e\left\{-\frac{\gamma}{\alpha} Q\left(x_{0}\right)\right\} \\
& \times \sum_{y \in \alpha^{-1} \widetilde{L}} P(y) e\left\{Q(y) \alpha z+2 B\left(y, x_{0}\right)\right\} \\
= & \frac{i^{m n}}{N(\alpha)^{m} \Phi(L)}\left(\frac{\gamma+1 / z}{\alpha}\right)^{m / 2+l} \\
& \times \sum_{y, x_{0} \in \widetilde{L} / \alpha L} e\left\{-\frac{\gamma}{\alpha} Q\left(x_{0}\right)+\frac{2}{\alpha} B\left(y, x_{0}\right)\right\} \theta(\alpha L, P, y ; z / \alpha) .
\end{aligned}
$$

Given any automorphy factor $j(A, z)$ for $A$, we know that

$$
\lim _{z \rightarrow i \infty} j(A, z)^{-m / 2-l} i^{m n}(\gamma+1 / z)^{m / 2+l}(-z)^{m / 2+l}=\varepsilon
$$

for some fourth root of unity $\varepsilon$ (depending on the choice of $j(A, z)$-note that the branch cut used for the square-root function is that described in Lemma 1.3.1 of [1]). We know that

$$
\lim _{z \rightarrow i \infty} \theta(\alpha L, P, y ; z / \alpha)=0,
$$

so $\lim _{z \rightarrow i \infty} \theta(L, P ; z) \mid A=0$. This proves

TheOREM 2. Given a nontrivial spherical harmonic $P$ relative to the lattice $L, \theta(L, P ; z)$ is a cusp form (and hence so is $\theta([L],[P] ; z)$ ).

3. The action of Hecke operators on $\theta([L],[P] ; z)$. Let $\mathcal{P}$ be a prime ideal not dividing $2 \mathcal{N}$. To describe the action of $T(\mathcal{P})$ or $T\left(\mathcal{P}^{2}\right)$ on $\theta([L],[P] ; z)$, we will need to understand the action of $S(\mathcal{P})$ on $\theta(L, P ; z)$. For $f \in \mathcal{M}_{k}\left(\Gamma_{0}(\mathcal{N}, N(L)), \chi_{L}\right)\left(\right.$ or $\left.\mathcal{M}_{k}\left(\widetilde{\Gamma}_{0}(\mathcal{N}, N(L)), \chi_{L}\right)\right)$ and $\mathcal{P}$ a prime 
ideal not dividing $\mathcal{N}$, we define $S(\mathcal{P})$ by

$$
f|S(\mathcal{P})=f| C \in \mathcal{M}_{k}\left(\Gamma_{0}\left(\mathcal{N}, \mathcal{P}^{2} N(L)\right), \chi_{L}\right)
$$

(when $k$ is integral) or

$$
f|S(\mathcal{P})=f|\left[C, N(\mathcal{P})^{-1 / 2} \frac{\theta_{\mathcal{I}_{L}}(C z)}{\theta_{\mathcal{P} \mathcal{I}_{L}}(z)}\right] \in \mathcal{M}_{k}\left(\widetilde{\Gamma}_{0}\left(\mathcal{N}, P^{2} N(L)\right), \chi_{L}\right)
$$

(when $k$ is half-integral); here

$$
C=\left(\begin{array}{ll}
a & b \\
c & d
\end{array}\right) \in\left(\begin{array}{cc}
\mathcal{P} & \mathcal{P}^{-1}(N(L))^{-1} \partial^{-1} \\
\mathcal{N} \mathcal{P} N(L) \partial & \mathcal{O}
\end{array}\right)
$$

with $d \equiv 1(\bmod \mathcal{N})$ and $a d-b c=1$. Now, $S(\mathcal{P})$ is an isomorphism, so we define $S\left(\mathcal{P}^{-1}\right)=S(\mathcal{P})^{-1}$, and we extend the definition of $S(*)$ multiplicatively so that $S(\mathcal{Q})$ is defined for all fractional ideals $\mathcal{Q}$ relatively prime to $2 \mathcal{N}$.

Let $\mathcal{I}$ be a fractional ideal and let $\mathcal{M}_{m / 2}\left(\Gamma, \chi_{L}\right)$ denote the space of Hilbert modular forms of weight $m / 2$ which transform with character $\chi_{L}$ under the group $\Gamma$. For $m$ even we define

$$
T(\mathcal{P}): \mathcal{M}_{m / 2}\left(\Gamma_{0}(\mathcal{N}, \mathcal{I}), \chi_{L}\right) \rightarrow \mathcal{M}_{m / 2}\left(\Gamma_{0}(\mathcal{N}, \mathcal{P} \mathcal{I}), \chi_{L}\right)
$$

by

$$
f\left|T(\mathcal{P})=N(\mathcal{P})^{m / 4-1} \sum_{A} f\right| A
$$

where $\{A\}$ is a complete set of coset representatives for

$$
\left(\Gamma_{1}(\mathcal{N}, \mathcal{I}) \cap \Gamma_{1}(\mathcal{N}, \mathcal{P} \mathcal{I})\right) \backslash \Gamma_{1}(\mathcal{N}, \mathcal{P} \mathcal{I}) .
$$

For $m$ odd we define

$$
T\left(\mathcal{P}^{2}\right): \mathcal{M}_{m / 2}\left(\widetilde{\Gamma}_{0}\left(\mathcal{N}, \mathcal{I}^{2}\right), \chi_{L}\right) \rightarrow \mathcal{M}_{m / 2}\left(\widetilde{\Gamma}_{0}\left(\mathcal{N}, \mathcal{P}^{2} \mathcal{I}^{2}\right), \chi_{L}\right)
$$

by

$$
f\left|T\left(\mathcal{P}^{2}\right)=N(\mathcal{P})^{m / 2-2} \sum_{\widetilde{A}} f\right| \widetilde{A}
$$

where $\{\widetilde{A}\}$ is a complete set of coset representatives for

$$
\left(\widetilde{\Gamma}_{1}\left(\mathcal{N}, \mathcal{I}^{2}\right) \cap \widetilde{\Gamma}_{1}\left(\mathcal{N}, \mathcal{P}^{2} \mathcal{I}^{2}\right)\right) \backslash \widetilde{\Gamma}_{1}\left(\mathcal{N}, \mathcal{P}^{2} \mathcal{I}^{2}\right) .
$$

$\left(\right.$ Here $\Gamma_{1}(\mathcal{N}, \mathcal{I})$ denotes the set of all elements of $\Gamma_{0}(\mathcal{N}, \mathcal{I})$ whose lower right entry is congruent to 1 modulo $\mathcal{N}$.)

Lemma. For $\mathcal{P}$ a prime ideal not dividing $2 \mathcal{N}$,

$$
\theta(L, P ; z) \mid S(\mathcal{P})=N(\mathcal{P})^{m / 2} \varepsilon_{L}(\mathcal{P}) \theta(\mathcal{P} L, P ; z)
$$


where

$$
\varepsilon_{L}(\mathcal{P})= \begin{cases}\left((-1)^{m / 2} \varepsilon_{\mathcal{P}} \mid \mathcal{P}\right) & \text { if } m \text { is even, } \\ \left(2 \varepsilon_{\mathcal{P}} \mid \mathcal{P}\right) & \text { if } m \text { is odd. }\end{cases}
$$

Here $\varepsilon_{\mathcal{P}} \in \mathcal{O}_{\mathcal{P}}^{\times}$such that $L_{\mathcal{P}} \simeq \varrho\left\langle 1, \ldots, 1, \varepsilon_{\mathcal{P}}\right\rangle$ for some $\varrho \in \mathbb{K}_{\mathcal{P}}$.

R e m a r k. When $m$ is even, the square class of $\varepsilon_{\mathcal{P}}$ is clearly independent of the choice of $\varrho$. When $m$ is odd, Lemma 3.1 of [10] shows that the square class of $\varepsilon_{\mathcal{P}}$ is independent of the choice of $\varrho$.

Proof. To prove this we essentially follow the arguments used to prove Proposition 6.1 of [7] and Proposition 3.3 of [10]. Take $C=\left(\begin{array}{ll}a & b \\ c & d\end{array}\right)$ as in the definition of $S(\mathcal{P})$. Then as in the proof of the transformation formula,

$$
\begin{aligned}
\theta(L, P & \left.; \frac{a z+b}{c z+d}\right) \\
= & \sum_{x_{0} \in L / d \mathcal{P} L} e\left\{\frac{b}{d} Q\left(x_{0}\right)\right\} \sum_{x \in d \mathcal{P} L} P\left(x+x_{0}\right) e\left\{\frac{Q\left(x+x_{0}\right)}{d(d / z+c)}\right\} \\
= & \frac{i^{m n / 2}}{\sqrt{\Phi(d \mathcal{P} L)}}(d(d / z+c))^{m / 2+l} \\
& \times \sum_{x_{0} \in L / d \mathcal{P} L} e\left\{\frac{b}{d} Q\left(x_{0}\right)\right\} \\
& \times \sum_{y \in d^{-1} \mathcal{P}-1} P(y) e\left\{-Q(y) d(d / z+c)+2 B\left(x_{0}, y\right)\right\} \\
= & \frac{i^{m n / 2}}{\sqrt{\Phi(d \mathcal{P} L)}}(d(d / z+c))^{m / 2+l} \\
& \times \sum_{x_{0} \in L / d \mathcal{P} L} e\left\{\frac{b}{d} Q\left(x_{0}\right)+\frac{2}{d} B\left(y, x_{0}\right)-\frac{c}{d} Q(y)\right\} \\
& \times N(d)^{-l} P(y) e\left\{-Q(y) \frac{1}{z}\right\} .
\end{aligned}
$$

Now, $\frac{b}{d} Q\left(x_{0}\right)+\frac{2}{d} B\left(y, x_{0}\right)-\frac{c}{d} Q(y) \equiv-\frac{c}{d} Q\left(b x_{0}+y\right)\left(\bmod 2 \partial^{-1}\right)$ so

$$
e\left\{\frac{b}{d} Q\left(x_{0}\right)+\frac{2}{d} B\left(y, x_{0}\right)-\frac{c}{d} Q(y)\right\}=e\left\{-\frac{c}{d} Q\left(b x_{0}+y\right)\right\} .
$$

Also, as $x_{0}$ runs over $L / d \mathcal{P} L, b x_{0}+y$ runs over $\mathcal{P}^{-1} \widetilde{L} / d \widetilde{L}$. Hence 


$$
\begin{aligned}
\theta(L, P ; & \left.\frac{a z+b}{c z+d}\right) \\
= & \frac{i^{m n / 2}}{\sqrt{\Phi(d \mathcal{P} L)}}(d(d / z+c))^{m / 2+l} N(d)^{-l} \\
& \times \sum_{\substack{x_{0} \in L / d \mathcal{P} L \\
y \in \mathcal{P}^{-1} \widetilde{L}}} e\left\{\frac{b}{d} Q\left(x_{0}\right)+\frac{2}{d} B\left(y, x_{0}\right)-\frac{c}{d} Q(y)\right\} P(y) e\left\{-Q(y) \frac{1}{z}\right\} \\
= & (d / z+c)^{m / 2+l} z^{m / 2+l} N(d)^{-m / 2} \sum_{x_{0} \in L / d \mathcal{P} L} e\left\{\frac{b}{d} Q\left(x_{0}\right)\right\} \theta(\mathcal{P} L, P ; z) \\
= & (d / z+c)^{m / 2+l} z^{m / 2+l} N(d)^{-m / 2} \sum_{x \in \mathcal{P} L / d \mathcal{P} L} e\left\{\frac{b}{d} Q(x)\right\} \\
& \times \sum_{y \in d L / d \mathcal{P} L} e\left\{\frac{b}{d} Q(y)\right\} \theta(\mathcal{P} L, P ; z) .
\end{aligned}
$$

When $m$ is even,

$$
N(d)^{-m / 2} \sum_{x \in \mathcal{P} L / d \mathcal{P} L} e\left\{\frac{b}{d} Q(x)\right\}=\chi_{L}(d)=1
$$

(since $d \equiv 1(\bmod \mathcal{N}))$ and

$$
\sum_{y \in d L / d \mathcal{P} L} e\left\{\frac{b}{d} Q(y)\right\}=N(\mathcal{P})^{m / 2} \varepsilon_{L}(\mathcal{P})
$$

(see Proposition 6.1 of [7]); hence

$$
\theta(L, P ; z) \mid S(\mathcal{P})=\varepsilon_{L}(\mathcal{P}) N(\mathcal{P})^{m / 2} \theta(\mathcal{P} L, P ; z) .
$$

When $m$ is odd, the above computation and the proof of Proposition 3.3 of [10] give us

$$
\theta(L, P ; z) \mid S(\mathcal{P})=\varepsilon_{L}(\mathcal{P}) N(\mathcal{P})^{m / 2} \theta(\mathcal{P} L, P ; z) .
$$

Now we can prove

TheOrem 3. Let $\mathcal{P}$ be a prime ideal not dividing $2 \mathcal{N}$.

(1) If $m$ is even then

$$
\begin{aligned}
\theta([L],[P] ; z) \mid T(\mathcal{P})= & N(\mathcal{P})^{m / 4} \sum_{\substack{x \in[L] \\
Q(x) \in \mathcal{P} \mathbf{n} L}}[P](x) e\{Q(x) z\} \\
& +\varepsilon_{L}(\mathcal{P}) N(\mathcal{P})^{3 m / 4-1} \sum_{x \in \mathcal{P}[L]}[P](x) e\{Q(x) z\}
\end{aligned}
$$


and

$$
\begin{aligned}
\theta([L],[P] ; z) \mid T(\mathcal{P})^{2}= & N(\mathcal{P})^{m / 2} \sum_{\substack{x \in[L] \\
Q(x) \in \mathcal{P}^{2} \mathbf{n} L}}[P](x) e\{Q(x) z\} \\
& +\varepsilon_{L}(\mathcal{P}) N(\mathcal{P})^{m-1} \sum_{\substack{x \in \mathcal{P}[L] \\
Q(x) \in \mathcal{P}^{3} \mathbf{n} L}}[P](x) e\{Q(x) z\} \\
& +\varepsilon_{L}(\mathcal{P}) N(\mathcal{P})^{m-1} \sum_{x \in \mathcal{P}[L]}[P](x) e\{Q(x) z\} \\
& +N(\mathcal{P})^{3 m / 2-2} \sum_{x \in \mathcal{P}^{2}[L]}[P](x) e\{Q(x) z\} .
\end{aligned}
$$

(2) If $m$ is odd then

$$
\begin{aligned}
\theta(L, P ; z) \mid T\left(\mathcal{P}^{2}\right)= & N(\mathcal{P})^{m / 2} \sum_{\substack{x \in L \\
Q(x) \in \mathcal{P}^{2} \mathbf{n} L}} P(x) e\{Q(x) z\} \\
& +N(\mathcal{P})^{m-3 / 2} \sum_{\substack{x \in \mathcal{P} L \\
Q(x) \notin \mathcal{P}^{3} \mathbf{n} L}} P(x) e\{Q(x) z\} \\
& +N(\mathcal{P})^{3 m / 2-2} \sum_{x \in \mathcal{P}^{2} L} P(x) e\{Q(x) z\}
\end{aligned}
$$

P r o of. Note that it suffices to consider the action of the Hecke operators on $\theta(L, P ; z)$, which we have seen is a Hilbert modular form. First consider the case where $m$ is even. Then

$$
\begin{aligned}
\theta(L, P ; z) \mid T(\mathcal{P})= & N(\mathcal{P})^{m / 4-1} \sum_{b} \theta(L, P ; z) \mid\left(\begin{array}{ll}
1 & b \\
0 & 1
\end{array}\right) \\
& +N(\mathcal{P})^{m / 4-1} \theta(L, P ; z) \mid S(\mathcal{P})
\end{aligned}
$$

and by the preceding lemma,

$$
\begin{aligned}
= & N(\mathcal{P})^{m / 4} \sum_{\substack{x \in L \\
Q(x) \in \mathcal{P} \mathbf{n} L}} P(x) e\{Q(x) z\} \\
& +\varepsilon_{L}(\mathcal{P}) N(\mathcal{P})^{3 m / 4-1} \sum_{x \in \mathcal{P} L} P(x) e\{Q(x) z\}
\end{aligned}
$$

where $b$ runs over $\mathcal{P}^{-1}(\mathbf{n} L)^{-1} \partial^{-1} /(\mathbf{n} L)^{-1} \partial^{-1}$.

Next we see that with $b$ running over $\mathcal{P}^{-2}(N(L))^{-1} \partial^{-1} /(N(L))^{-1} \partial^{-1}$, $\beta$ running over $\mathcal{P}^{-2}(N(L))^{-1} \partial^{-1} / \mathcal{P}^{-1}(N(L))^{-1} \partial^{-1}$ and $\gamma$ running over $\mathcal{P}^{-1}(N(L))^{-1} \partial^{-1} /(N(L))^{-1} \partial^{-1}$, we have 


$$
\begin{aligned}
N(\mathcal{P})^{2-} & m / 2 \theta(L, P ; z) \mid T(\mathcal{P})^{2} \\
= & \sum_{b} \theta(L, P ; z)\left|\left(\begin{array}{ll}
1 & b \\
0 & 1
\end{array}\right)+\sum_{\beta} \theta(L, P ; z)\right| S(\mathcal{P}) \mid\left(\begin{array}{cc}
1 & \beta \\
0 & 1
\end{array}\right) \\
& \quad+\sum_{\gamma} \theta(L, P ; z)\left|\left(\begin{array}{ll}
1 & \gamma \\
0 & 1
\end{array}\right)\right| S(\mathcal{P})+\theta(L, P ; z)|S(\mathcal{P})| S(\mathcal{P}) \\
= & N(\mathcal{P})^{2} \sum_{\substack{x \in L \\
Q(x) \in \mathcal{P}^{2} N(L)}} P(x) e\{Q(x) z\}+\varepsilon_{L}(\mathcal{P}) N(\mathcal{P})^{m / 2+1} \theta(\mathcal{P} L, P ; z) \\
& +N(\mathcal{P})^{m} \theta\left(\mathcal{P}^{2} L, P ; z\right)+\sum_{\gamma^{\prime}} \theta(L, P ; z)|S(\mathcal{P})|\left(\begin{array}{cc}
1 & \gamma^{\prime} \\
0 & 1
\end{array}\right)
\end{aligned}
$$

where $\gamma^{\prime}$ runs over $\mathcal{P}^{-3}(N(L))^{-1} \partial^{-1} /(N(L))^{-1} \partial^{-1}$. Part (1) of the theorem now follows.

Now suppose $m$ is odd. By Lemma 2.2 of [10],

$$
\begin{aligned}
& N(\mathcal{P})^{-m / 2+2} \theta(L, P ; z) \mid T\left(\mathcal{P}^{2}\right) \\
& =\sum_{b} \theta(L, P ; z) \mid\left[\left(\begin{array}{ll}
1 & b \\
0 & 1
\end{array}\right), 1\right] \\
& \quad+\sum_{\beta} \theta(L, P ; z)|S(\mathcal{P})|\left[\left(\begin{array}{cc}
1 & \beta \\
0 & 1
\end{array}\right), N(\mathcal{P})^{1 / 2}\left(\sum_{\alpha \in \mathcal{P I}_{L} / \mathcal{P}^{2} \mathcal{I}_{L}} e\left\{-2 \beta \alpha^{2}\right\}\right)^{-1}\right] \\
& \quad+\theta(L, P ; z) \mid S\left(\mathcal{P}^{2}\right)
\end{aligned}
$$

where $b$ runs over $\mathcal{P}^{-2} N(L)^{-1} \partial^{-1} / N(L)^{-1} \partial^{-1}$ and $\beta$ runs over

$$
\left(\mathcal{P}^{-3} N(L)^{-1} \partial^{-1} / \mathcal{P}^{-2} N(L)^{-1} \partial^{-1}\right)^{\times}
$$

So to prove part (2) of the theorem, we need to evaluate the middle term. As shown in $\S 3$ of [7],

So

$$
\left(\sum_{\alpha} e\left\{-2 \beta \alpha^{2}\right\}\right)^{2}=(-1 \mid \mathcal{P}) N(\mathcal{P})
$$

$$
\begin{aligned}
\sum_{\beta} \theta & (L, P ; z)|S(\mathcal{P})|\left[\left(\begin{array}{cc}
1 & \beta \\
0 & 1
\end{array}\right), N(\mathcal{P})^{1 / 2}\left(\sum_{\alpha \in \mathcal{P} \mathcal{I}_{L} / \mathcal{P}^{2} \mathcal{I}_{L}} e\left\{-2 \beta \alpha^{2}\right\}\right)^{-1}\right] \\
= & \varepsilon_{L}(\mathcal{P})(-1 \mid \mathcal{P})^{(m-1) / 2} N(\mathcal{P})^{(m-1) / 2} \sum_{\beta} \theta(\mathcal{P} L, P ; z+\beta) \sum_{\alpha} e\left\{-2 \beta \alpha^{2}\right\} \\
= & \varepsilon_{L}(\mathcal{P})(-1 \mid \mathcal{P})^{(m-1) / 2} N(\mathcal{P})^{(m-1) / 2} \\
& \times \sum_{x \in \mathcal{P} L} P(x) e\{Q(x) z\} \sum_{\alpha, \beta} e\{Q(x) \beta\} e\left\{-2 \beta \alpha^{2}\right\} .
\end{aligned}
$$


Note that as shown in the proof of Lemma 3.1 of [10], $\operatorname{ord}_{\mathcal{P}} \mathbf{n} L$ must be even (since $\mathcal{P}$ does not divide $\mathcal{N}$ ) and hence $\operatorname{ord}_{\mathcal{P}} \mathbf{n} L=\operatorname{ord}_{\mathcal{P}} N(L)$. Thus $Q(x) \in \mathcal{P}^{3} \mathbf{n} L$ if and only if $Q(x) \in \mathcal{P}^{3} N(L)$. Suppose $Q(x) \in \mathcal{P}^{3} \mathbf{n} L$; then $e\{Q(x) \beta\}=1$. Furthermore, taking $\varrho \in \mathcal{P}^{-1} \partial^{-1}-\partial^{-1}$, the techniques used in $\S 3$ of $[7]$ give us

$$
\begin{aligned}
\sum_{\alpha, \beta} e\{Q(x) \beta\} e\left\{-2 \beta \alpha^{2}\right\} & =\sum_{\substack{\alpha, \beta \in \mathcal{O} / \mathcal{P} \\
\beta \notin \mathcal{P}}} e\left\{-2 \varrho \beta \alpha^{2}\right\}=\sum_{\substack{\alpha, \beta \in \mathcal{O} / \mathcal{P} \\
\beta \notin \mathcal{P}}}(\alpha \mid \mathcal{P}) e\{-2 \varrho \beta \alpha\} \\
& =\sum_{\alpha, \beta \in \mathcal{O} / \mathcal{P}}(\alpha \beta \mid \mathcal{P}) e\{-2 \varrho \alpha\}=0
\end{aligned}
$$

(since $\left.\sum_{\beta \in \mathcal{O} / \mathcal{P}}(\beta \mid \mathcal{P})=0\right)$.

So now suppose $Q(x) \notin \mathcal{P}^{3} \mathbf{n} L$. A similar computation shows that when we take $\varrho \in \mathcal{P}^{-1} \partial^{-1}-\partial^{-1}$ and $\mu \in \mathcal{P}^{-1} \mathcal{I}_{L}^{-1}-\mathcal{I}_{L}^{-1}$ we have

$$
\begin{aligned}
\sum_{\alpha, \beta} e\{Q(x) \beta\} e\left\{-2 \beta \alpha^{2}\right\} & =\sum_{\substack{\alpha, \beta \in \mathcal{O} / \mathcal{P} \\
\beta \notin \mathcal{P}}} e\left\{Q(x) \mu^{2} \varrho \beta\right\} e\left\{-2 \varrho \beta \alpha^{2}\right\} \\
& =\sum_{\beta \in \mathcal{O} / \mathcal{P}} e\left\{Q(x) \mu^{2} \varrho \beta\right\} \sum_{\alpha \in \mathcal{O} / \mathcal{P}}(-\beta \mid \mathcal{P}) e\left\{2 \varrho \alpha^{2}\right\} \\
& =(-1 \mid \mathcal{P}) \sum_{\beta \in \mathcal{O} / \mathcal{P}} e\left\{Q(x) \mu^{2} \varrho \beta^{2}\right\} \sum_{\alpha \in \mathcal{O} / \mathcal{P}} e\left\{2 \varrho \alpha^{2}\right\} \\
& =\left(-2 Q(x) \mu^{2} \mid \mathcal{P}\right) \sum_{\alpha, \beta \in \mathcal{O} / \mathcal{P}} e\left\{2 \varrho\left(\alpha^{2}+\beta^{2}\right)\right\} \\
& =\left(2 Q(x) \mu^{2} \mid \mathcal{P}\right) N(\mathcal{P}) .
\end{aligned}
$$

Now, as argued at the end of the proof of Theorem 7.4 of [7],

$$
(-1 \mid \mathcal{P})^{(m-1) / 2} \varepsilon_{L}(\mathcal{P})\left(2 Q(x) \mu^{2} \mid \mathcal{P}\right)=1
$$

for $x \in \mathcal{P} L$ with $Q(x) \notin \mathcal{P}^{3} \mathbf{n} L$. Using the preceding lemma now yields part (2) of the theorem.

To count the vectors of $L$ the number of times indicated in the theorem, we use special sublattices of $L$ which we define as follows.

Definitions. Given a prime ideal $\mathcal{P}$ not dividing $2 \mathcal{N}$, we say a lattice $L^{\prime}$ is a $\mathcal{P}$-sublattice of $L$ if $\mathcal{P} L \subseteq L^{\prime} \subseteq L$ and $L^{\prime} / \mathcal{P} L$ is a maximal totally isotropic subspace of $L / \mathcal{P} L$. A lattice $L^{\prime \prime}$ is a $\mathcal{P}^{2}$-sublattice of $L$ if $L^{\prime \prime}$ is a $\mathcal{P}$-sublattice of some $\mathcal{P}$-sublattice $L^{\prime}$ of $L$ such that $\operatorname{dim} L^{\prime \prime} /\left(\mathcal{P} L \cap L^{\prime \prime}\right)=$ $\operatorname{dim} L^{\prime} / \mathcal{P} L$.

With these definitions and Propositions 7.2 and 7.3 of [7] (where we count occurrences of different vectors of $L$ in $\mathcal{P}$ - and $\mathcal{P}^{2}$-sublattices of $L$ ), we get a generalization of the Eichler Commutation Relation: 
Corollary. Let $\mathcal{P}$ be a prime ideal not dividing $2 \mathcal{N}$.

(1) For $m$ even and $\varepsilon_{L}(\mathcal{P})=1$,

$$
\theta(L, P ; z) \mid T(\mathcal{P})=N(\mathcal{P})^{m / 4} \lambda^{-1} \sum_{L^{\prime}} \theta\left(L^{\prime}, P ; z\right)
$$

where $\lambda=\left(N(\mathcal{P})^{m / 2-1}+1\right) \ldots\left(N(\mathcal{P})^{0}+1\right)$ and the sum is over all $\mathcal{P}$ sublattices $L^{\prime}$ of $L$.

(2) For $m$ even and $\varepsilon_{L}(\mathcal{P})=-1$,

$$
\begin{aligned}
\theta(L, P ; z) \mid T(\mathcal{P})^{2} & =N(\mathcal{P})^{m / 2} \kappa^{-1} \sum_{L^{\prime \prime}} \theta\left(L^{\prime \prime}, P ; z\right) \\
& +N(\mathcal{P})^{m / 2}\left(1-N(\mathcal{P})^{m / 2-2}-2 N(\mathcal{P})^{m / 2-1}\right) \theta(\mathcal{P} L, P ; z)
\end{aligned}
$$

where $\kappa=N(\mathcal{P})^{m / 2-3} \ldots N(\mathcal{P})^{0}\left(N(\mathcal{P})^{m / 2-1}+1\right) \ldots\left(N(\mathcal{P})^{2}+1\right)$ and the sum is over all $\mathcal{P}^{2}$-sublattices $L^{\prime \prime}$ of $L$.

(3) For $m$ odd,

$$
\begin{aligned}
& \theta(L, P ; z) \mid T\left(\mathcal{P}^{2}\right) \\
& =\left\{\begin{array}{cc}
\left(N(\mathcal{P})^{-1 / 2}+N(\mathcal{P})^{1 / 2}\right) \theta(L, P ; z) & \text { if } m=1, \\
N(\mathcal{P})^{m / 2} \kappa^{-1} \sum_{L^{\prime \prime}} \theta\left(L^{\prime \prime}, P ; z\right) & \\
\quad+\left(N(\mathcal{P})^{m / 2}-N(\mathcal{P})^{m-3 / 2}\right) \theta(\mathcal{P} L, P ; z) & \text { if } m>1,
\end{array}\right.
\end{aligned}
$$

where $\kappa=N(\mathcal{P})^{(m-5) / 2} \ldots N(\mathcal{P})^{0}\left(N(\mathcal{P})^{(m-3) / 2}+1\right) \ldots(N(\mathcal{P})+1)$ and the sum is over all $\mathcal{P}^{2}$-sublattices $L^{\prime \prime}$ of $L$.

4. Eigenforms and linear dependence relations. We can now follow the constructions of eigenforms given in [9] and [10].

Let gen $L$ denote the genus of $L$. For $m$ even, let fam $^{+} L$ denote the nuclear family of $L$ (as defined in [9]); so a lattice $L^{\prime}$ is in the nuclear family if there exists some totally positive unit $u$ such that $L_{\mathcal{P}}^{\prime} \simeq L_{\mathcal{P}}^{u}$ for all primes $\mathcal{P}$. We also let fam $L$ denote the family of $L$; a lattice $L^{\prime}$ is in the family of $L$ if $L^{\prime}$ is a lattice on $V^{\alpha}$ for some $\alpha \gg 0$ with $(\alpha, 2 \mathcal{N})=1$ such that for every prime $\mathcal{P}$ there is a local unit $u_{\mathcal{P}}$ giving us $L_{\mathcal{P}}^{\prime} \simeq L_{\mathcal{P}}^{u_{\mathcal{P}}}$. We set

$$
\theta(\operatorname{gen}[L],[P] ; z)=\theta\left(\operatorname{fam}^{+} L,[P] ; z\right)=\sum_{L^{\prime} \in \operatorname{gen} L} \frac{1}{o\left(L^{\prime}\right)} \theta\left(\left[L^{\prime}\right],[P] ; z\right)
$$

where $o\left(L^{\prime}\right)$ denotes the order of the orthogonal group of $L^{\prime}$. Notice that for $m$ even,

$$
\theta(\operatorname{gen}[L],[P] ; z)=\sum_{L^{\prime} \in \mathrm{fam}^{+} L^{+}} \frac{1}{o\left(L^{\prime}\right)} \theta(L,[P] ; z) .
$$

Now the corollary to Theorem 3 together with the counting arguments used to prove Theorem 3.6 of [9] give us the 
Lemma. Let $\mathcal{P}$ be a prime ideal not dividing $2 \mathcal{N}$.

(1) If $m$ is even and $\varepsilon_{L}(\mathcal{P})=1$ then

$$
\theta(\operatorname{gen}[L],[P] ; z) \mid T(\mathcal{P})=N(\mathcal{P})^{m / 4}\left(N(\mathcal{P})^{m / 2-1}+1\right) \theta(\operatorname{gen}[K],[P] ; z)
$$

where $K$ is any $\mathcal{P}$-sublattice of $L$.

(2) If $m$ is even but $\varepsilon_{L}(\mathcal{P})=-1$ then

$$
\theta(\operatorname{gen}[L],[P] ; z) \mid T(\mathcal{P})^{2}=N(\mathcal{P})^{m / 2}\left(N(\mathcal{P})^{m / 2-1}-1\right)^{2} \theta(\operatorname{gen}[\mathcal{P} L],[P] ; z) .
$$

(3) If $m$ is odd then

$$
\theta(\text { gen } L, P ; z) \mid T\left(\mathcal{P}^{2}\right)=N(\mathcal{P})^{m / 2}\left(N(\mathcal{P})^{m-2}+1\right) \theta(\text { gen } \mathcal{P} L, P ; z) .
$$

The operators in the above lemma together with the operators $S(\mathcal{P})$ $(\mathcal{P}$ not dividing $2 \mathcal{N})$ generate an algebra $\mathcal{T}^{L}$ which is a subalgebra of the Hecke algebra $\mathcal{T}$. However, $\mathcal{T}^{L}$ does not act on $\mathcal{M}_{m / 2+l}\left(\Gamma_{0}(\mathcal{N}, N(L)), \chi_{L}\right)$; we let $\mathcal{T}_{0}$ denote the subalgebra of $\mathcal{T}$ which does act on this space (cf. [8], [9]). We also let $\mathcal{T}_{0}^{L}=\mathcal{T}^{L} \cap \mathcal{T}_{0}$. For $m$ odd, the above lemma implies that $\theta$ (gen $L, P ; z)$ is an eigenform for $\mathcal{T}_{0}^{L}$ (provided it is nonzero).

Consider the case where $m$ is even. Let $\mathcal{Q}_{1}, \ldots, \mathcal{Q}_{t}$ be the bad primes for $L$ as defined in [9] (so for each $i, 1 \leq i \leq t$, there is a local unit $u_{i}$ such that $L_{\mathcal{Q}_{i}}^{u_{i}} \nsucceq L_{\mathcal{Q}_{i}}$ ). Assume here that these bad primes are nondyadic; in the case where some bad prime is dyadic the construction of our eigenforms is more complicated and is described in $\S 1$ of [10]. Set

$$
U_{L}=\left\{\left(\left(u \mid \mathcal{Q}_{1}\right), \ldots,\left(u \mid \mathcal{Q}_{t}\right)\right): u \in \mathcal{U}^{+}\right\} .
$$

We associate the nuclear families within fam $L$ with cosets in $\{ \pm 1\}^{t} / U_{L}$ as follows. Let gens $\mathcal{T}^{L}$ be the commutative monoid consisting of all (finite) products of the operators

$$
\left\{T(\mathcal{P}): \varepsilon_{L}(\mathcal{P})=1\right\} \cup\left\{T(\mathcal{P})^{2}: \varepsilon_{L}(\mathcal{P})=-1\right\} \cup\{S(\mathcal{I})\}
$$

where it is understood that $\mathcal{P}$ is a prime ideal not dividing $2 \mathcal{N}$ and $\mathcal{I}$ is a fractional ideal relatively prime to $2 \mathcal{N}$. Then as vector spaces, $\mathcal{T}^{L}$ is generated by gens $\mathcal{T}^{L}$ and $\mathcal{T}_{0}^{L}$ is generated by gens $\mathcal{T}_{0}^{L}=\mathcal{T}_{0} \cap$ gens $\mathcal{T}^{L}$. Take $T \in$ gens $\mathcal{T}_{0}^{L}$. Thus

$$
T=T\left(\mathcal{P}_{1}\right) \ldots T\left(\mathcal{P}_{l}\right) T\left(\mathcal{P}_{l+1}\right)^{2} \ldots T\left(\mathcal{P}_{l+s}\right)^{2} S(\mathcal{I})
$$

where the $\mathcal{P}_{i}$ are primes (not necessarily distinct) such that $\varepsilon_{L}\left(\mathcal{P}_{i}\right)=1$ for $1 \leq i \leq l, \varepsilon_{L}\left(\mathcal{P}_{i}\right)=-1$ for $i>l$, and $\alpha \mathcal{P}_{1} \ldots \mathcal{P}_{l} \mathcal{P}_{l+1}^{2} \ldots \mathcal{P}_{l+s}^{2} \mathcal{I}^{2}=\mathcal{O}$ for some $\alpha \gg 0$. Let $K=K_{l+s} \subset \ldots \subset K_{1} \subset K_{0}=L$ be lattices

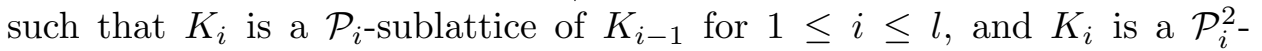
sublattice of $K_{i-1}$ for $i>l$. Then repeated use of the lemma and the corollary of Section 3 shows that $\theta(\operatorname{gen}[L],[P] ; z) \mid T=\lambda_{T} \theta\left(\operatorname{gen}\left[\mathcal{I} K^{\alpha}\right],[P] ; z\right)$ (where $\lambda_{T} \neq 0$ depends only on $T$ ); we associate $\operatorname{fam}^{+} \mathcal{I} K^{\alpha}=\operatorname{gen}\left[\mathcal{I} K^{\alpha}\right]$ 
with the coset $\left(\left(\alpha \mid \mathcal{Q}_{1}\right), \ldots,\left(\alpha \mid \mathcal{Q}_{t}\right)\right) U_{L}$. In this way each nuclear family in fam $L$ is associated with some $\operatorname{coset}\left(\left(\beta \mid \mathcal{Q}_{1}\right), \ldots,\left(\beta \mid \mathcal{Q}_{t}\right)\right) U_{L}$; let $L_{\beta}$ be a lattice in the nuclear family associated with this coset. Then just as when $L_{\beta}=L, \theta\left(\operatorname{gen}\left[L_{\beta}\right],[P] ; z\right) \mid T=\lambda_{T} \theta\left(\operatorname{gen}\left[L_{\alpha \beta}\right],[P] ; z\right)$ with $\lambda_{T}$ as above.

Now we can use $\left\{\theta\left(\operatorname{gen}\left[L_{\beta}\right],[P] ; z\right)\right\}$ to construct "eigenforms" for $\mathcal{T}_{0}^{L}$. (Note that these will not be eigenforms if they are 0 , and we will show that at least in some cases the forms we construct are 0.) Let $\mathcal{A}$ be an integral ideal dividing $\mathcal{Q}_{1} \ldots \mathcal{Q}_{t}$. Set

$$
E_{\mathcal{A}}(z)=\sum_{\beta}(\beta \mid \mathcal{A}) \theta\left(\operatorname{gen}\left[L_{\beta}\right],[P] ; z\right)
$$

where $\beta$ varies so that gen $\left[L_{\beta}\right]=\operatorname{fam}^{+} L_{\beta}$ varies over the nuclear families within fam $L$; then for $T$ as above,

$$
\begin{aligned}
E_{\mathcal{A}}(z) \mid T & =\lambda_{T} \sum_{\beta}(\beta \mid \mathcal{A}) \theta\left(\operatorname{gen}\left[L_{\alpha \beta}\right],[P] ; z\right) \\
& =\lambda_{T}(\alpha \mid \mathcal{A}) \sum_{\beta}(\alpha \beta \mid \mathcal{A}) \theta\left(\operatorname{gen}\left[L_{\alpha \beta}\right],[P] ; z\right)=\lambda_{T}(\alpha \mid \mathcal{A}) E_{\mathcal{A}}(z) .
\end{aligned}
$$

Hence the nonzero $E_{\mathcal{A}}(z)$ are $\mathcal{T}_{0}^{L}$-eigenforms. Note that if $E_{\mathcal{A}}(z)$ and $E_{\mathcal{A}^{\prime}}(z)$ are nonzero and $(\alpha \mid \mathcal{A}) \neq\left(\alpha \mid \mathcal{A}^{\prime}\right)$ for some $\alpha$ such that $L_{\alpha} \in$ fam $L$, then $E_{\mathcal{A}}(z)$ and $E_{\mathcal{A}^{\prime}}(z)$ are linearly independent.

We now lift these $\mathcal{T}_{0}^{L}$-eigenforms to $\mathcal{T}^{L}$-eigenforms as follows. Let $\mathcal{I}_{1}, \ldots, \mathcal{I}_{h^{\prime}}$ represent the distinct (nonstrict) ideal classes; take ideals $\mathcal{J}_{1}, \ldots, \mathcal{J}_{h / h^{\prime}}$ such that the $\mathcal{J}_{\eta} \mathcal{I}_{\mu}^{2}$ represent the distinct strict ideal classes. (Note that the $\mathcal{I}_{\mu}^{2}$ necessarily lie in distinct strict classes, for if $\mathcal{I}^{2}=\beta \mathcal{O}$ for $\beta \gg 0$ then by the Global Square Theorem $\beta$ is necessarily the square of some element of $\mathbb{K}^{\times}$.) Without loss of generality, assume $\mathcal{J}_{1}=N(L)$ and $\mathcal{I}_{1}=\mathcal{O}$, and assume that the $\mathcal{I}_{\sigma}$ are relatively prime to $2 \mathcal{N}$. Thus $\theta(\operatorname{gen}[L],[P] ; z)$ is an element of $\mathcal{M}_{m / 2+l}\left(\Gamma_{0}\left(\mathcal{N}, \mathcal{J}_{1} \mathcal{I}_{1}^{2}\right), \chi_{L}\right)$ (if $m$ is even) or $\mathcal{M}_{m / 2+l}\left(\widetilde{\Gamma}_{0}\left(\mathcal{N}, \mathcal{J}_{1} \mathcal{I}_{1}^{2}\right), \chi_{L}\right)$ (if $m$ is odd). Let

$$
\mathcal{M}_{m / 2+l}\left(\mathcal{N}, \chi_{L}\right)= \begin{cases}\prod_{\eta, \mu} \mathcal{M}_{m / 2+l}\left(\Gamma_{0}\left(\mathcal{N}, \mathcal{J}_{\eta} \mathcal{I}_{\mu}^{2}\right), \chi_{L}\right) & \text { if } m \text { is even } \\ \prod_{\mu} \mathcal{M}_{m / 2+l}\left(\widetilde{\Gamma}_{0}\left(\mathcal{N}, \mathcal{J}_{1} \mathcal{I}_{\mu}^{2}\right), \chi_{L}\right) & \text { if } m \text { is odd }\end{cases}
$$

and let

$$
\begin{array}{r}
\mathcal{M}_{m / 2+l}(\mathcal{N}, \chi)=\left\{F \in \mathcal{M}_{m / 2+l}\left(\mathcal{N}, \chi_{L}\right): F \mid S(\mathcal{Q})=\chi^{*}(\mathcal{Q}) F\right. \\
\text { for all ideals } \mathcal{Q},(\mathcal{Q}, 2 \mathcal{N})=1\} .
\end{array}
$$

Here $\chi$ denotes any Hecke character extending $\chi_{L}$ (see [8], [6]). For later convenience we assume that $\chi$ is chosen such that for $\mathcal{P}$ not dividing $2 \mathcal{N}$, $\chi^{*}(\mathcal{P})=\varepsilon_{L}(\mathcal{P})($ cf. [9]). 
First consider the case where $m$ is odd. Define $\Theta(\operatorname{gen}[L],[P] ; z)$ to be the element of $\mathcal{M}_{m / 2+l}\left(\mathcal{N}, \chi_{L}\right)$ whose $\mu$-component is $N\left(\mathcal{I}_{\mu}\right)^{m / 2} \times$ $\theta\left(\operatorname{gen}\left[\mathcal{I}_{\mu} L\right],[P] ; z\right)$. Then by the lemma of Section $3, \Theta(\operatorname{gen}[L],[P] ; z) \in$ $\mathcal{M}_{m / 2+l}(\mathcal{N}, \chi)$, and

$$
\Theta(\operatorname{gen}[L],[P] ; z) \mid T\left(\mathcal{P}^{2}\right)=\left(N(\mathcal{P})^{m-2}+1\right) \Theta(\operatorname{gen}[L],[P] ; z)
$$

for all prime ideals $\mathcal{P}$ not dividing $\mathcal{N}$.

Now consider the case where $m$ is even; in this case we basically follow the procedure described in [8], but in view of what we know about the $E_{\mathcal{A}}$ we can simplify this procedure a bit.

Let $[\mathcal{I}]$ denote the complex of the strict ideal class of $\mathcal{I}$ (so $[\mathcal{I}]=\left\{\mathcal{I}^{\prime}\right.$ : $\mathcal{I}^{\prime}=\alpha \mathcal{I}^{2}$ for some $\alpha \gg 0$ and some fractional ideal $\left.\left.\mathcal{J}\right\}\right)$. For notational convenience, we let $T_{0}\left(\mathcal{P}_{1}^{e_{1}} \ldots \mathcal{P}_{r}^{e_{r}}\right)=T\left(\mathcal{P}_{1}\right)^{e_{1}} \ldots T\left(\mathcal{P}_{r}\right)^{e_{r}}$ where $\mathcal{P}_{1}, \ldots, \mathcal{P}_{r}$ are prime ideals. Set

$$
C\left(E_{\mathcal{A}}\right)=\left\{[\mathcal{I}]: T_{0}(\mathcal{Q}) \in \mathcal{T}^{L} \text { for some } \mathcal{Q} \in[\mathcal{I}]\right\} ;
$$

when $\mathcal{Q} \in[\mathcal{I}]$ such that $T_{0}(\mathcal{Q}) \in \mathcal{T}^{L}$ we say $\mathcal{Q}$ witnesses $[\mathcal{I}] \in C\left(E_{\mathcal{A}}\right)$. (To compare this definition of $C\left(E_{\mathcal{A}}\right)$ to that given in Section 2 of [8], we simply remark that by the lemmas of this and the preceding sections, $E_{\mathcal{A}} \mid T_{0}\left(\mathcal{Q}^{2}\right) S\left(\mathcal{Q}^{-1}\right) \neq 0$ whenever $E_{\mathcal{A}} \neq 0$ and $T_{0}(\mathcal{Q}) \in \mathcal{T}^{L}$; thus we see that as required in [8], $E_{\mathcal{A}} \mid T_{0}(\mathcal{Q})=0$ whenever $E_{\mathcal{A}} \mid T_{0}\left(\mathcal{Q}^{2}\right) S\left(\mathcal{Q}^{-1}\right)=0$.) Notice that $C\left(E_{\mathcal{A}}\right)$ is a group in which every element has order 1 or 2 . Let $\left[\mathcal{Q}_{1}\right], \ldots,\left[\mathcal{Q}_{s}\right]$ generate $C\left(E_{\mathcal{A}}\right)$ such that $\mathcal{Q}_{j}$ witnesses $\left[\mathcal{Q}_{j}\right] \in C\left(E_{\mathcal{A}}\right)$. Choose $c_{j} \in \mathbb{C}^{\times}$such that

$$
E_{\mathcal{A}} \mid T_{0}\left(\mathcal{Q}_{j}^{2}\right) S\left(\mathcal{Q}_{j}^{-1}\right)=c_{j}^{2} \bar{\chi}^{*}\left(\mathcal{Q}_{j}\right) E_{\mathcal{A}}
$$

(Recall $T_{0}\left(\mathcal{Q}_{j}^{2}\right) S\left(\mathcal{Q}_{j}^{-1}\right) \in \mathcal{T}_{0}^{L}$.) We define $F_{\mathcal{A}} \in \mathcal{M}_{m / 2+l}(\mathcal{N}, \chi)$ as follows. Set the $(1,1)$-component of $F_{\mathcal{A}}$ equal to $E_{\mathcal{A}}$. If $\left[\mathcal{J}_{1}^{-1} \mathcal{J}_{\mu}\right] \notin C\left(E_{\mathcal{A}}\right)$ then set the $(\mu, \eta)$-component of $F_{\mathcal{A}}$ equal to 0 for all $\eta, 1 \leq \eta \leq h^{\prime}$. Now suppose $\left[\mathcal{J}_{1}^{-1} \mathcal{J}_{\mu}\right] \in C\left(E_{\mathcal{A}}\right)$. Choose $\eta$ such that $\mathcal{J}_{\mu} \mathcal{I}_{\eta}^{2} \sim \mathcal{Q}_{1}^{t_{1}} \ldots \mathcal{Q}_{s}^{t_{s}} \mathcal{J}_{1}$ where $t_{j}=0$ or 1 and $\mathcal{I} \sim \mathcal{J}$ means $\mathcal{I}$ and $\mathcal{J}$ are in the same strict ideal class. Set the $(\mu, \eta)$-component of $F_{\mathcal{A}}$ equal to

$$
\frac{1}{c_{1}^{t_{1}} \ldots c_{s}^{t_{s}}} E_{\mathcal{A}} \mid T_{0}\left(\mathcal{Q}_{1}^{t_{1}} \ldots \mathcal{Q}_{s}^{t_{s}}\right) \text {. }
$$

(Note that the exponents $t_{j}$ are uniquely determined by the complex of $\mathcal{J}_{\mu}$; also note that $E_{\mathcal{A}} \mid T_{0}\left(\mathcal{Q}_{1}^{t_{1}} \ldots \mathcal{Q}_{s}^{t_{s}}\right) \in \mathcal{M}_{m / 2+l}\left(\Gamma_{0}\left(\mathcal{N}, \mathcal{J}_{\mu} \mathcal{I}_{\eta}\right), \chi_{L}\right)$.) For each $\sigma \neq \eta, 1 \leq \sigma \leq h^{\prime}$, choose $\mathcal{I}$ relatively prime to $2 \mathcal{N}$ such that $\mathcal{I}_{\eta}^{2} \mathcal{I}^{2} \sim \mathcal{I}_{\sigma}^{2}$; set the $(\mu, \sigma)$-component of $F_{\mathcal{A}}$ equal to

$$
\bar{\chi}^{*}(\mathcal{I}) F_{\mathcal{A}}^{\mu \eta} \mid S(\mathcal{I}) \in \mathcal{M}_{m / 2+l}\left(\Gamma_{0}\left(\mathcal{N}, \mathcal{J}_{\mu} \mathcal{I}_{\sigma}\right), \chi_{L}\right)
$$

where $F_{\mathcal{A}}^{\mu \eta}$ denotes the $(\mu, \eta)$-component of $F_{\mathcal{A}}$. Notice that since $F_{\mathcal{A}}^{\mu \eta} \mid S(a \mathcal{O})$ 
$=\chi^{*}(a \mathcal{O}) F_{\mathcal{A}}^{\mu \eta}$ for any $a \in \mathbb{K}^{\times}$, the definition of $F_{\mathcal{A}}^{\mu \eta}$ is independent of the choice of $\mathcal{I}$.

It is clear from the construction of $F_{\mathcal{A}}$ that $F_{\mathcal{A}} \mid S(\mathcal{Q})=\chi^{*}(\mathcal{Q}) F_{\mathcal{A}}$ for all fractional ideals $\mathcal{Q}$ relatively prime to $2 \mathcal{N}$. Thus $F_{\mathcal{A}} \in \mathcal{M}_{m / 2+l}(\mathcal{N}, \chi)$. To show $F_{\mathcal{A}}$ is a $\mathcal{T}^{L}$-eigenform (provided $E_{\mathcal{A}} \neq 0$ and hence $F_{\mathcal{A}} \neq 0$ ), we take $\mathcal{P}$ to be a prime ideal not dividing $2 \mathcal{N}$. Then the lemmas of this and the previous sections show us that

$$
E_{\mathcal{A}} \mid T(\mathcal{P})^{2} S\left(\mathcal{P}^{-1}\right)=\varepsilon_{L}(\mathcal{P})\left(N(\mathcal{P})^{m / 2-1}+\varepsilon_{L}(\mathcal{P})\right)^{2} E_{\mathcal{A}} ;
$$

from the construction of $F_{\mathcal{A}}$, we find that

$$
\begin{aligned}
F_{\mathcal{A}} \mid T(\mathcal{P})^{2} & =\varepsilon_{L}(\mathcal{P}) \chi^{*}(\mathcal{P})\left(N(\mathcal{P})^{m / 2-1}+\varepsilon_{L}(\mathcal{P})\right)^{2} F_{\mathcal{A}} \\
& =\left(N(\mathcal{P})^{m / 2-1}+\varepsilon_{L}(\mathcal{P})\right)^{2} F_{\mathcal{A}}
\end{aligned}
$$

$\left(\right.$ recall that we chose $\chi$ so that $\left.\chi^{*}(\mathcal{P})=\varepsilon_{L}(\mathcal{P})\right)$. Suppose $\varepsilon_{L}(\mathcal{P})=1$. Choose $t_{j}=0$ or 1 and $\mathcal{I}$ a fractional ideal (relatively prime to $2 \mathcal{N}$ ) so that $\mathcal{P} \sim \mathcal{Q}_{1}^{-t_{1}} \ldots \mathcal{Q}_{s}^{-t_{s}} \mathcal{I}^{-2}$. Then $T=T_{0}\left(\mathcal{P} \mathcal{Q}_{1}^{t_{1}} \ldots \mathcal{Q}_{s}^{t_{s}}\right) S(\mathcal{I}) \in \mathcal{T}_{0}^{L}$. Thus $E_{\mathcal{A}} \mid T=\lambda_{T} E_{\mathcal{A}}$ and consequently for every $\mu$ and $\sigma$ we have $F_{\mathcal{A}}^{\mu \sigma} \mid T=\lambda_{T} F_{\mathcal{A}}^{\mu \sigma}$. Also,

$$
F_{\mathcal{A}} \mid T_{0}\left(\mathcal{Q}_{j}^{2}\right)=c_{j}^{2} F_{\mathcal{A}}
$$

so

$$
\begin{aligned}
& F_{\mathcal{A}}^{\mu \sigma} \mid T_{0}\left(\mathcal{Q}_{j}\right) \\
& =\frac{\bar{\chi}^{*}(\mathcal{I})}{c_{1}^{t_{1}} \ldots c_{s}^{t_{s}}} E_{\mathcal{A}} \mid T_{0}\left(\mathcal{Q}_{1}^{t_{1}} \ldots \mathcal{Q}_{s}^{t_{s}}\right) T_{0}\left(\mathcal{Q}_{j}\right) S(\mathcal{I}) \\
& = \begin{cases}\frac{\bar{\chi}^{*}\left(\mathcal{Q}_{j}\right) \bar{\chi}^{*}(\mathcal{I})}{c_{1}^{t_{1}} \ldots c_{s}^{t_{s}}} c_{j}^{2} E_{\mathcal{A}} \mid T_{0}\left(\mathcal{Q}_{1}^{t_{1}} \ldots \mathcal{Q}_{j}^{0} \ldots \mathcal{Q}_{s}^{t_{s}}\right) S\left(\mathcal{Q}_{j} \mathcal{I}\right) & \text { if } t_{j}=1 \\
\frac{\bar{\chi}^{*}(\mathcal{I})}{c_{1}^{t_{1}} \ldots c_{s}^{t_{s}}} E_{\mathcal{A}} \mid T_{0}\left(\mathcal{Q}_{1}^{t_{1}} \ldots \mathcal{Q}_{j}^{1} \ldots \mathcal{Q}_{s}^{t_{s}}\right) S(\mathcal{I}) & \text { if } t_{j}=0\end{cases} \\
& = \begin{cases}c_{j} \frac{\bar{\chi}^{*}\left(\mathcal{Q}_{j} \mathcal{I}\right)}{c_{1}^{t_{1}} \ldots c_{j}^{0} \ldots c_{s}^{t_{s}}} E_{\mathcal{A}} \mid T_{0}\left(\mathcal{Q}_{1}^{t_{1}} \ldots \mathcal{Q}_{j}^{0} \ldots \mathcal{Q}_{s}^{t_{s}}\right) S\left(\mathcal{Q}_{j} \mathcal{I}\right) & \text { if } t_{j}=1 \\
c_{j} \frac{\bar{\chi}^{*}(\mathcal{I})}{c_{1}^{t_{1}} \ldots c_{j}^{1} \ldots c_{s}^{t_{s}}} E_{\mathcal{A}} \mid T_{0}\left(\mathcal{Q}_{1}^{t_{1}} \ldots \mathcal{Q}_{j}^{1} \ldots \mathcal{Q}_{s}^{t_{s}}\right) S(\mathcal{I}) & \text { if } t_{j}=0\end{cases} \\
& =
\end{aligned}
$$

for appropriate $\mu^{\prime}$ and $\sigma^{\prime}$. Hence

$$
\lambda_{T} F_{\mathcal{A}}=F_{\mathcal{A}}\left|T=c_{1}^{t_{1}} \ldots c_{s}^{t_{s}} \chi^{*}(\mathcal{I}) F_{\mathcal{A}}\right| T(\mathcal{P}) .
$$

So if $E_{\mathcal{A}} \neq 0, F_{\mathcal{A}}$ is a $\mathcal{T}^{L}$-eigenform. In fact, for $\mathcal{P}$ a prime ideal not dividing $2 \mathcal{N}$

$$
F_{\mathcal{A}}(z) \mid T(\mathcal{P})= \pm\left(N(\mathcal{P})^{m / 2-1}+1\right) F_{\mathcal{A}}(z)
$$


when $\varepsilon_{L}(\mathcal{P})=1$, and

$$
F_{\mathcal{A}}(z) \mid T(\mathcal{P})^{2}=\left(N(\mathcal{P})^{m / 2-1}-1\right)^{2} F_{\mathcal{A}}(z)
$$

when $\varepsilon_{L}(\mathcal{P})=-1$. (Note that when $\varepsilon_{L}(\mathcal{P})=1$, we can determine the sign of the eigenvalue by using the methods of [9].)

We know that for an integral weight cuspidal $\mathcal{T}$-eigenform of weight $m / 2+l$, its eigenvalue under $T(\mathcal{P})$ is on the order of $N(\mathcal{P})^{(m+2 l) / 4}$ (and so its eigenvalues under $T(\mathcal{P})^{2}$ are on the order of $\left.N(\mathcal{P})^{(m+2 l) / 2}\right)$. We also know that a cuspidal $T^{L}$-eigenform is a linear combination of $\mathcal{T}$-eigenforms (see [6]). So unless $l \geq m / 2-2$, the $F_{\mathcal{A}}(z)$ are necessarily 0 . Letting $\beta$ vary as before and letting $\mathcal{A}$ vary over the divisors of $\mathcal{Q}_{1} \ldots \mathcal{Q}_{t}$, we see that

$$
\sum_{\mathcal{A}} E_{\mathcal{A}}(z)=\sum_{\beta}\left(\sum_{\mathcal{A}}(\beta \mid \mathcal{A})\right) \theta\left(\operatorname{gen}\left[L_{\beta}\right],[P] ; z\right) .
$$

If $\left(\beta \mid \mathcal{Q}_{j}\right)=1$ for all $j$ then $\operatorname{gen}\left[L_{\beta}\right]=\operatorname{gen}[L]$. If $\left(\beta \mid \mathcal{Q}_{j}\right)=-1$ for some $j$, then $(\beta \mid \mathcal{A})=1$ for exactly half the ideals $\mathcal{A}$ dividing $\mathcal{Q}_{1} \ldots \mathcal{Q}_{t}$. Thus

$$
\sum_{\mathcal{A}}(\beta \mid \mathcal{A})= \begin{cases}2^{t} & \text { if gen }\left[L_{\beta}\right]=\operatorname{gen}[L], \\ 0 & \text { otherwise }\end{cases}
$$

and so $\sum_{\mathcal{A}} E_{\mathcal{A}}(z)=2^{t} \theta(\operatorname{gen}[L],[P] ; z)$. This means we have

THEOREM 4. If $m$ is even and $l$, the degree of the spherical harmonic $P \neq 1$, is less than $m / 2-2$ then $\theta(\operatorname{gen}[L],[P] ; z)=0$ and so

$$
\theta([L],[P] ; z)=o(L) \sum_{\substack{L^{\prime} \in \operatorname{gen} L \\\left[L^{\prime}\right] \neq[L]}} \frac{1}{o\left(L^{\prime}\right)} \theta\left(\left[L^{\prime}\right],[P] ; z\right) .
$$

Remarks. 1. Although the estimates on eigenvalues are not sufficient to show this, we suspect this theorem holds for all $l>0$.

2. When $\mathbb{K}=\mathbb{Q}$, the Shimura lift yields bounds on the magnitude of eigenvalues for half-integral weight modular forms, so the theorem can be extended to include the case of $m$ odd and $\mathbb{K}=\mathbb{Q}$.

3. Let $\mathbb{K}=\mathbb{Q}$ and let $L$ be a lattice with an integral positive definite quadratic form $Q$. If $P$ is an indefinite quadratic form such that its associated matrix relative to a $\mathbb{Z}$-basis for $L$ has trace 0 then we can use $P$ as a degree 2 spherical harmonic. In this case the above theorem shows that for rank $L>6$,

$$
\sum_{\substack{x \in \bigcup_{Q(x)=n} \operatorname{gen} L \\ Q(x)=0}} P(x)=0
$$

for every integer $n$. (Here $\bigcup$ gen $L$ denotes the union of the lattices in the genus of $L$ relative to the positive definite form $Q$.) 


\section{References}

[1] A. N. Andrianov, Quadratic Forms and Hecke Operators, Springer, New York 1987.

[2] M. Eichler, The Basis Problem for Modular Forms and the Traces of the Hecke Operators, Lecture Notes in Math. 320, Springer, 1973.

[3] - On theta functions of real algebraic number fields, Acta Arith. 33 (1977), 269292.

[4] H. Hijikata, A. K. Pizer and T. R. Shemanske, The basis problem for modular forms on $\Gamma_{0}(N)$, Mem. Amer. Math. Soc. 418 (1989).

[5] O. T. O'Meara, Introduction to Quadratic Forms, Springer, New York 1973.

[6] T. R. Shemanske and L. H. Walling, Twists of Hilbert modular forms, Trans. Amer. Math. Soc., to appear.

[7] L. H. Walling, Hecke operators on theta series attached to lattices of arbitrary rank, Acta Arith. 54 (1990), 213-240.

[8] —, On lifting Hecke eigenforms, Trans. Amer. Math. Soc. 328 (1991), 881-896.

[9] - , Hecke eigenforms and representation numbers of quadratic forms, Pacific J. Math. 151 (1991), 179-200.

[10] - Hecke eigenforms and representation numbers of arbitrary rank lattices, ibid., 156 (1992), 371-394.

[11] - An arithmetic version of Siegel's representation formula, to appear.

[12] - A remark on differences of theta series, J. Number Theory, to appear.

DEPARTMENT OF MATHEMATICS

UNIVERSITY OF COLORADO AT BOULDER

BOULDER, COLORADO 80309

U.S.A.

E-mail: WALLING@EUCLID.COLORADO.EDU 